\title{
RECONSTRUCTION OF 3D DENSE CARDIAC MOTION FROM TAGGED MR SEQUENCES
}

\author{
Hsun-Hsien Chang ${ }^{1} \quad$ José M.F. Moura ${ }^{1} \quad$ Yijen L. Wu ${ }^{2} \quad$ Kazuya Sato ${ }^{2}$ Chien Ho $^{2}$ \\ ${ }^{1}$ Department of Electrical and Computer Engineering \\ ${ }^{2}$ Pittsburgh NMR Center for Biomedical Research \\ Carnegie Mellon University, \\ Pittsburgh, Pennsylvania, USA
}

\begin{abstract}
This paper develops an energy minimization algorithm to reconstruct the 3D motion of transplanted hearts of small animals (rats) from tagged magnetic resonance (MR) sequences. We describe the heart by a layered aggregate of thin oriented elastic fibers. We use the orientation of myocardial fibers to develop a local dense motion of the heart. This dense model is fit to the tagged MRI data by minimizing an energy functional with two terms: the first term is the external energy, derived from matching the image intensities on the fibers across two consecutive frames; the second term is the fibers' internal energy, derived from biomechanics analysis. This paper illustrates the application of the approach to a set of cardiac MR sequences containing four slices of a transplanted rat heart.
\end{abstract}

\section{INTRODUCTION}

Studying the left ventricle (LV) is most important in cardiac function analysis. Cardiologists have widely used the left ventricle's global parameters, such as stroke volume and electrocardiogram, to identify the severity of patients' cardiac diseases. However, these global parameters are not good indicators of unhealthy hearts at early stages of disease. Cardiologists are increasingly interested in identifying the local cardiac malfunction, for example, the local infarction and ischemia, so that they can start the treatment of sick hearts as early as possible.

Cardiac magnetic resonance tagging [1] provides an opportunity for cardiologists to understand the local cardiac malfunction. The tagged MR images display the myocardium with lines superimposed, as shown in Fig. 1. When the heart moves, the tag lines deform consistently with the heart. Sun et al. [2] have developed a method that propagates the motions of tag lines to all pixels of the

This work was supported by the National Institute of Health under Grants R01EB/AI-00318 and P4EB001977.

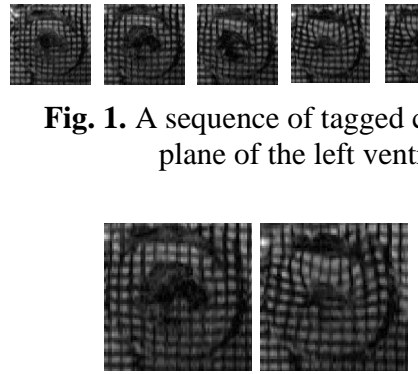

(a) Two frames at end-systole
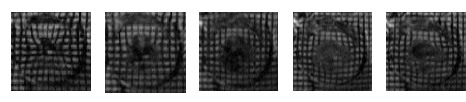

Fig. 2. 2D dense displacements of the left ventricle.

heart and then provides a dense representation for the motions in each 2D slice of the heart, see Fig. 2.

At common MRI resolutions, heart slices are 10 to 15 pixels apart. To reconstruct dense 3D motions, we propose here to use a cardiac fiber based model $[3,4]$. The heart is represented by layers of thin elastic fibers that stretch and compress through the cardiac cycle. The fibers are described as a concatenation of many small segments. We use this model in an energy minimization approach to reconstruct the 3D motion of the left ventricle.

In this paper, Section 2 describes the myocardial fiber structure. Section 3 introduces a motion model for the fibers. Section 4 develops the energy functional to be minimized. Section 5 presents our experimental results in reconstructing the 3D motion of the left ventricle of a rat heart. Finally, Section 6 concludes the paper.

\section{MYOCARDIAL STRUCTURE}

The cardiac muscle cells of the myocardium are arranged in layers that are tightly bounded together and completely encircle the blood-filled chambers, as shown in Fig. 3(a). Streeter [3, 4] quantitatively analyzed the myocardial fiber orientations. He excised a block of a canine myocardium, and cut the parallel cross sections from endocardial to epicardial surfaces through this block. His studies concluded that the fibers are almost tangential to the endocar- 


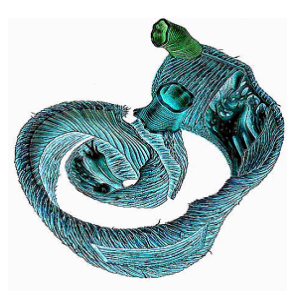

(a)

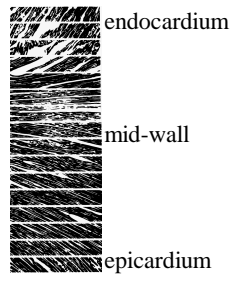

(b)
Fig. 3. The structure and orientation of the fibers of the heart.

dial and epicardial surfaces. Furthermore, the fiber orientations vary from $-60^{\circ}$ at epicardium to $+60^{\circ}$ at endocardium. This pattern is shown in Fig. 3(b).

Physiologists usually view the myocardial fibers as small pieces of cylinders and take into account fiber orientations in cardiac structural analysis [5]. This viewpoint is usually ignored in image analysis. In this paper, we use the orientations and cylindrical shapes of the myocardial fibers to reconstruct the left ventricular motion from tagged MR sequences.

We model the left ventricle as a thick, flexible cylinder with the height of $h$ and with the outer radius of $\rho$, when the heart fully extends at the end-diastole, as shown in Fig. 4. This cylinder is composed of many fibers. Each fiber is one pixel thick and extends from the bottom slice to the top slice at an orientation $\eta$. From Streeter's studies, $\eta=-60^{\circ}$ at epicardium, so that the length of a fiber is approximately 60 pixels, assuming four slices and 10-pixel slice thickness.

We now discuss the generation of the fiber. The location of a fiber on the epicardial surface can be described as a helix, see Fig. 4:

$$
x=\rho \cdot \cos \theta, y=\rho \cdot \sin \theta, \text { and } z=\beta \cdot \theta,
$$

where $\theta$ is the rotation angle in the $x-y$ plane, i.e., the slice plane, and $\beta$ is the coefficient controlling how fast the $z$ component of the helix climbs. From prior knowledge, the fiber follows the known orientation $\eta$; this can be used to determine the parameter $\beta$. With reference to Fig. 4 , for a small rotation on the slice plane $\Delta \theta$,

$$
\tan \eta=\frac{\beta \cdot \Delta \theta}{\rho \cdot \Delta \theta} .
$$

It follows that $\beta=\rho \cdot \tan \eta$. We assume $\eta$ known and obtain $\rho$ from MRI data; so, $\beta$ can be estimated by (2).

\section{CONTINUUM MECHANICS MODEL}

We now develop a motion model for our fiber based heart. Since the left ventricle suffers small deformations through the cardiac cycle, an infinitesimal deformation model is an appropriate description for the movement. Based on this assumption, we divide a long fiber into a series of small segments each 1 pixel long.

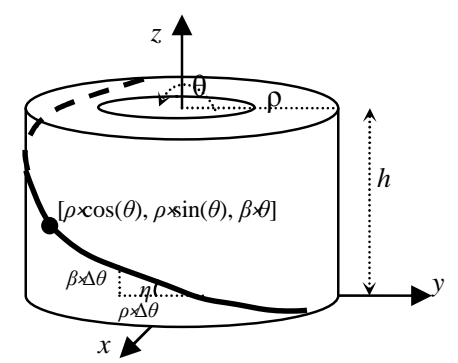

Fig. 4. A thick cylinder models the shape of the left ventricle, and a helix describes the location of a myocardial fiber on the surface of the left ventricle.

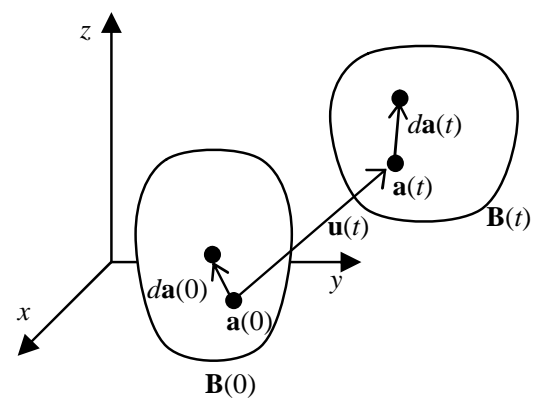

Fig. 5. The deformation of a left ventricle.

\subsection{The Jacobian matrix}

The left ventricle in 3D space is denoted by $\mathbf{B}(t)$. Initially it is $\mathbf{B}(0)$ and deforms into $\mathbf{B}(t)$ at time $t$, as shown in Fig. 5. A pixel $\mathbf{a}(0)$ within $\mathbf{B}(0)$ moves to $\mathbf{a}(t)$ within $\mathbf{B}(t)$. The location of $\mathbf{a}(t)$ is related to its initial condition by

$$
\mathbf{a}(t)=\mathbf{u}(t)+\mathbf{a}(0),
$$

where $\mathbf{u}(t)$ is the displacement. Let $\mathbf{a}(0)+d \mathbf{a}(0)$ be a neighboring point of $\mathbf{a}(0)$ that moves to $\mathbf{a}(t)+d \mathbf{a}(t)$ according to the equation, [6],

$$
d \mathbf{a}(t)=\frac{\partial \mathbf{a}(t)}{\partial \mathbf{a}(0)} d \mathbf{a}(0) .
$$

The Jacobian matrix is defined by

$$
\mathbf{F}(t)=\frac{\partial \mathbf{a}(t)}{\partial \mathbf{a}(0)}=\left[\begin{array}{lll}
\frac{\partial a_{1}(t)}{\partial a_{1}(0)} & \frac{\partial a_{1}(t)}{\partial a_{2}(0)} & \frac{\partial a_{1}(t)}{\partial a_{3}(0)} \\
\frac{\partial a_{2}(t)}{\partial a_{1}(0)} & \frac{\partial a_{2}(t)}{\partial a_{2}(0)} & \frac{\partial a_{2}(t)}{\partial a_{3}(0)} \\
\frac{\partial a_{3}(t)}{\partial a_{1}(0)} & \frac{\partial a_{3}(t)}{\partial a_{2}(0)} & \frac{\partial a_{3}(t)}{\partial a_{3}(0)}
\end{array}\right],
$$

which is also known as the deformation gradient matrix. At $t=0, \mathbf{F}(0)=\mathbf{I}$. By plugging (3) into (5) for each entry, the Jacobian has components, [6],

$$
F_{i j}(t)=\frac{\partial a_{i}(t)}{\partial a_{j}(0)}=\frac{\partial\left(u_{i}(t)+a_{i}(0)\right)}{\partial a_{j}(0)}=\frac{\partial u_{i}(t)}{\partial a_{j}(0)}+\delta_{i j},
$$


where $\delta_{i j}$ is the Kronecker symbol that is 1 when $i=j$ and is 0 otherwise. From (6), the Jacobian can be rewritten as

$$
\mathbf{F}(t)=\mathbf{I}+d \mathbf{F}(t)=\mathbf{I}+\left[\begin{array}{lll}
\frac{\partial u_{1}(t)}{\partial a_{1}(0)} & \frac{\partial u_{1}(t)}{\partial a_{2}(0)} & \frac{\partial u_{1}(t)}{\partial a_{3}(0)} \\
\frac{\partial u_{2}(t)}{\partial a_{1}(0)} & \frac{\partial u_{2}(t)}{\partial a_{2}(0)} & \frac{\partial u_{2}(t)}{\partial a_{3}(0)} \\
\frac{\partial u_{3}(t)}{\partial a_{1}(0)} & \frac{\partial u_{3}(t)}{\partial a_{2}(0)} & \frac{\partial u_{3}(t)}{\partial a_{3}(0)}
\end{array}\right] .
$$

\subsection{The strain energy}

When a flexible rod is bent, the work applied is stored as strain energy. By measuring the displacements of the points of the rod, we can calculate the strain energy accumulated by the rod. For finite deformations, the strain tensor $\mathbf{E}$ is defined as

$$
\mathbf{E} \triangleq \frac{1}{2}\left(\mathbf{F}^{\mathrm{T}} \mathbf{F}-\mathbf{I}\right) .
$$

Using infinitesimal analysis, in continuum mechanics, the strain tensor is usually approximated by the small strain tensor

$$
\mathbf{S} \approx \frac{1}{2}\left(\mathbf{F}+\mathbf{F}^{\mathrm{T}}\right)-\mathbf{I} .
$$

Approximation (9) follows by replacing (7) into (8) and ignoring the second order terms. Since $\mathbf{S}$ is symmetric, we vectorize the six entries located on the upper triangle of $\mathbf{S}$ as the vector $\mathbf{s}$ :

$$
\mathbf{s}=\left[S_{11}, S_{22}, S_{33}, S_{12}, S_{13}, S_{23}\right]^{T} .
$$

The segment $d \mathbf{a}(0)$ in Fig. 5 is assumed small. When the small segment deforms to $d \mathbf{a}(t)$, the strain energy $e$ it accumulates is computed by a linear elasticity model [6]

$$
e=\mathbf{s}^{\mathrm{T}} \mathbf{C s},
$$

where $\mathbf{C}$ is a $6 \times 6$ matrix describing the anisotropic properties of the myocardial fibers and takes the form

$$
\mathbf{C}^{-1}=\left[\begin{array}{cccccc}
\frac{1}{E_{\perp}} & \frac{-v_{\perp}}{E_{\perp}} & \frac{-v_{\|}}{E_{\|}} & 0 & 0 & 0 \\
\frac{-v_{\perp}}{E_{\perp}} & \frac{1}{E_{\perp}} & \frac{-v_{\|}}{E_{\|}} & 0 & 0 & 0 \\
\frac{-v_{\|} E_{\|}}{E_{\perp}} & \frac{-v_{\|} E_{\|}}{E_{\perp}} & \frac{1}{E_{\|}} & 0 & 0 & 0 \\
0 & 0 & 0 & \frac{2\left(1+v_{\perp}\right)}{E_{\perp}} & 0 & 0 \\
0 & 0 & 0 & 0 & \frac{2\left(1+v_{\|}\right)}{E \|} & 0 \\
0 & 0 & 0 & 0 & 0 & \frac{2\left(1+v_{\|}\right)}{E \|}
\end{array}\right],
$$

where $E_{\|}$is the along-fiber stiffness, $E_{\perp}$ the cross-fiber stiffness, and $v_{\|}$and $v_{\perp}$ are the corresponding Poisson's ratios. Replacing (7) into (9), it follows that the small strain tensor $\mathbf{S}$ is a function of the displacement $\mathbf{u}$. Hence, the strain energy $e$ is also a function of $\mathbf{u}$.

In our fiber model, we divide an elastic long fiber into many small segments, as shown in Fig. 6. The fiber's strain energy $\varepsilon$ is given by the sum of the strain energy of each segment:

$$
\varepsilon=\sum_{\text {all segnents }} \mathbf{s}^{\mathrm{T}} \mathbf{C s} .
$$

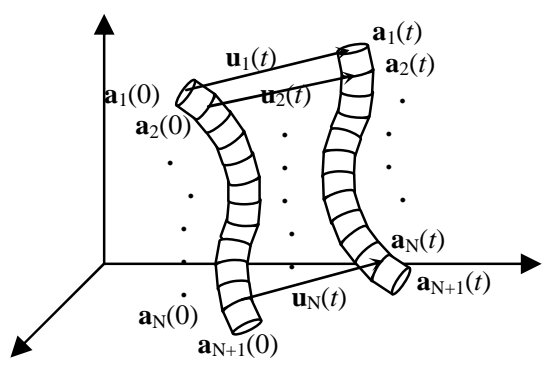

Fig. 6. The deformation of a myocardial fiber.

It follows that the strain energy $\varepsilon$ is a function of the displacements of all segments. With reference to Fig. 6, we vectorize the displacements $\mathbf{u}_{n}$ of $\mathrm{N}$ segments of the fiber into the vector $\mathbf{U}=\left[\mathbf{u}_{1}^{\mathrm{T}}, \mathbf{u}_{2}^{\mathrm{T}}, \cdots, \mathbf{u}_{N}^{\mathrm{T}}\right]^{\mathrm{T}}$; hence, the strain energy $\varepsilon$ is now a function of $\mathbf{U}$.

\section{ENERGY FUNCTIONAL}

We now define the energy functional $E(\mathbf{U})$ that will be minimized to derive the dense displacement field $\mathbf{U}$. We first introduce the notation used to describe the deformation of a fiber, see Fig. 6. A fiber has $\mathrm{N}$ segments. The endpoints of a segment are referred to as nodes. The node, $\mathbf{a}_{n}(0)$ for $n \leq \mathrm{N}$, its displacement, $\mathbf{u}_{n}(t)=\mathbf{a}_{n}(t)-\mathbf{a}_{n}(0)$, and its small segment, $\mathbf{a}_{n+1}(0)-\mathbf{a}_{n}(0)$, play the same roles as $\mathbf{a}(0), \mathbf{u}(t)$ and $d \mathbf{a}(0)$ in Section 3, respectively. We define the energy functional

$$
\mathrm{E}(\mathbf{U})=\gamma_{1} \mathrm{E}_{1}(\mathbf{U})+\gamma_{2} \mathrm{E}_{2}(\mathbf{U}),
$$

as the sum of two terms, where $\gamma_{i}$ are the weightings. The term $E_{1}(\mathbf{U})$ is the external energy that accounts for the mismatch of the fiber image intensities across two consecutive frames. The term $E_{2}(\mathbf{U})$ is the internal energy that corresponds to the strain energy described in Section 3.2.

External Energy: The intensity of a pixel $(i, j, k)$ in a fiber at time $t$ is labeled by $I_{i j k}(t)$. We vectorize all pixel intensities of the fiber at time $t$ by the vector $\mathbf{I}(t)$. The external energy is then given by

$$
\mathrm{E}_{1}(\mathbf{U})=\|\mathbf{I}(t)-\mathbf{I}(t+1)\|^{2} .
$$

Internal Energy: Because a long fiber is represented by a series of tiny segments, we can apply the linear elasticity model, (11), to each segment. From (13), the fiber's internal energy $E_{2}(\mathbf{U})$ is

$$
\mathrm{E}_{2}(\mathbf{U})=\sum_{n=1}^{N} \varepsilon_{n}=\sum_{n=1}^{N} \mathbf{s}_{n}^{\mathrm{T}} \mathbf{C} \mathbf{s}_{n} .
$$

The two terms of the energy functional attempt to strike a balance between matching the intensities across frames and the mechanics of the fiber. If $E_{1}(\mathbf{U})$ were not 
present, minimization of $E_{2}(\mathbf{U})$ alone would penalize any displacement and enforce the fiber to move back to its initial location.

Displacement Constraints: Our 3D image domain consists of four transversal MR slices. We fill the space between a pair of MR slices by virtual slices obtained by interpolation. On the other hand, on the four MR slices, the movements of the tag lines can be accurately measured [2]. We treat the motions on the tag lines as a constraint in the energy functional. These constraints are considered via Lagrange multiplier $\lambda_{\alpha}$. The energy functional becomes the Hamiltonian

$$
\mathrm{E}\left(\mathbf{U}, \lambda_{\alpha}\right)=\gamma_{1} \mathrm{E}_{1}(\mathbf{U})+\gamma_{2} \mathrm{E}_{2}(\mathbf{U})+\lambda_{\alpha}\left(\mathbf{U}^{\mathrm{T}} \boldsymbol{\Theta}_{\alpha}-\mathbf{u}_{\alpha}\right),
$$

where $\boldsymbol{\Theta}_{\alpha}=[0, \cdots, 0,1,1,1,0, \cdots, 0]^{\mathrm{T}}$ has 1 's only at the entries of the location of the node $\alpha$ on the tag line.

\section{EXPERIMENTAL RESULTS}

Transplanted rats were studied by using heterotopic working hearts. 4 transversal slices are taken to cover the heart at 10 time frames per cardiac cycle. All MRI scans were performed on a Bruker AVANCE DRX 4.7-T system. Each image has $256 \times 256$ pixels. The segmentation, tag line detection, and 2D displacement estimation are carried out by using the algorithms developed in [2].

A left ventricle structure with 3 layers, as shown in Fig. 7(a), is generated according to the method described in Section 2. To initialize the minimization of (17), we assume that the myocardial fibers are oriented according to the prediction in $[3,4]$. Since we only use 3 layers, the orientations of the fibers are taken to be from $-45^{\circ}$ at epicardium to $+45^{\circ}$ at endocardium. These are shown in Figs. 7(b) ... 7(d).

We apply the method in Section 4 to reconstruct the 3D left ventricle. Figs. 8(a) and 8(b) show the systolic and diastolic 3D motions of the epicardial left ventricle, respectively. The motions depicted in the figures are quite realistic. We see that the basal left ventricle (on the top) has more pronounced motions at the end-systole than at the end-diastole as it should be. The figures also illustrate well how the myocardial fibers deform through the cardiac cycle. The results show not only the fiber displacements, but also the myocardial rotation, stretch, and compression that are very useful in clinic studies.

\section{CONCLUSIONS}

In this paper, we develop a myocardial fiber based model to describe the left ventricle and use this model to reconstruct accurately the 3D motion of the left ventricle. The

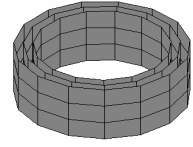

(a) The cylindrical layers of LV

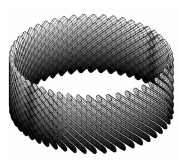

(b) The epicardial layer

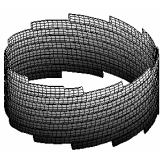

(c) The midwall layer

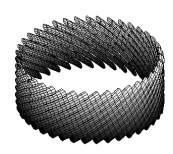

(d) The endocardial layer
Fig. 7. The initialization of the left ventricle.

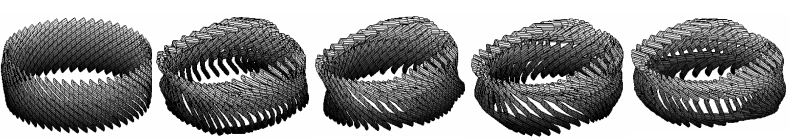

(a) systole (from left to right)

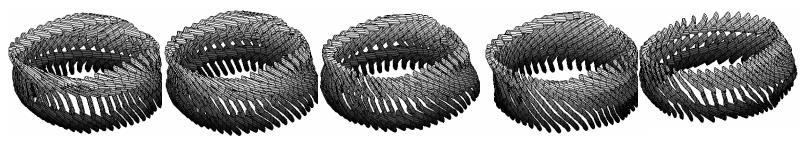

(b) diastole (from left to right)

Fig. 8. The epicardial deformation of the left ventricle.

reconstruction is achieved by minimizing an energy functional that combines the fibers' external energy, obtained from the images, with an internal energy derived from biomechanics. Experimental results with real tagged MR sequences demonstrate that the fiber model based reconstruction is appropriate to study the myocardial rotation, stretch, and compression.

\section{REFERENCES}

[1] L. Axel and L. Dougherty, "Heart wall motion: Improved method of spatial modulation of magnetization for MR imaging," Radiol., vol. 172, pp. 349-350, 1989.

[2] Y. Sun, Y.L. Wu, K. Sato, C. Ho, and J.M.F. Moura, "Dense motion estimation in tagged MRI sequence," in Proc. 11th Annu. Conf. Intl. Soc. Mag. Reson. Med., Toronto, Canada, p. 778, 2003

[3] D.D. Streeter and W.T. Hanna, "Engineering mechanics for successive states in canine left ventricular myocardium: I. Cavity and wall geometry," Circ. Res., vol. 33, pp. 639-655, 1973.

[4] D.D. Streeter, "Gross morphology and fiber geometry in the heart," in Handbook of Physiology Volume 1: the Cardiovascular System, R.M. Berne et al., Ed., American Physiological Society, Baltimore, pp. 61-112, 1979.

[5] P.J. Hunter, B.H. Smaill, P.M.F. Nielsen, and I.J. LeGrice, "A mathematical model of cardiac anatomy," in Computational Biology of the Heart, A.V. Panfilov and A.V. Holden, Ed., John Wiley \& Sons, New York, pp. 171-215, 1997

[6] X. Papademetris, A.J. Sinusas, and J.S. Duncan, "Computational platforms for integrated cardiac image analysis," in Measurement of Cardiac Deformations from MRI: Physical and Mathematical Models, A.A. Amini and J.L. Prince, Ed., Kluwer Academic, The Netherlands, pp. 289-313, 2001 